\title{
Cosmological Galaxy Evolution with Superbubble Feedback II: The Limits of Supernovae
}

\author{
B. W. Keller ${ }^{1 \star}$, J. Wadsley ${ }^{1}$, H. M. P. Couchman ${ }^{1}$ \\ ${ }^{1}$ Department of Physics and Astronomy, McMaster University, Hamilton, Ontario, L8S 4M1, Canada
}

14 November 2017

\begin{abstract}
We explore when supernovae can (and cannot) regulate the star formation and bulge growth in galaxies based on a sample of 18 simulated galaxies. The simulations include key physics such as evaporation and conduction, neglected in prior work, and required to correctly model superbubbles resulting from stellar feedback. We show that for galaxies with virial masses $>10^{12} \mathrm{M}_{\odot}$, supernovae alone cannot prevent excessive star formation. This failure occurs due to a shutdown of galactic winds, with wind mass loadings falling from $\eta \sim 10$ to $\eta<1$. In more massive systems, this transfer of baryons to the circumgalactic medium falters earlier on and the galaxies diverge significantly from observed galaxy scaling relations and morphologies. The decreasing efficiency is simply due to a deepening potential well preventing gas escape. This implies that non-supernova feedback mechanisms must become dominant for galaxies with stellar masses greater than $\sim 4 \times 10^{10} \mathrm{M}_{\odot}$. The runaway growth of the central stellar bulge, strongly linked to black hole growth, suggests that feedback from active galactic nuclei is the probable mechanism. Below this mass, supernovae alone are able to produce a realistic stellar mass fraction, star formation history and disc morphology.
\end{abstract}

Key words: - galaxies:formation - galaxies:evolution - galaxies:ISM - conduction cosmology:theory

\section{INTRODUCTION}

Stellar feedback plays many roles in galaxies. Simply regulating the star formation rate within the interstellar medium (ISM) is not enough to produce a realistic galaxy population. The reduced baryon fractions seen in galaxies requires the expulsion of that gas (Mathews \& Baker 1971; Larson 1974). Matching observed scaling relations, cosmic star formation histories, the stellar mass-metallicity regulation, and the stellar mass function all require the ejection of gas from a galaxy in large-scale outflows (Dekel \& Silk 1986; Finlator \& Davé 2008; Erb 2008; Peeples \& Shankar 2011; Marasco et al. 2012). Both abundance matching (Behroozi et al. 2013; Moster et al. 2013) and gravitational lensing studies (Hudson et al. 2015) find that no galaxies convert more than $\sim 25 \%$ of their inital baryonic mass into stars. The most straight forward explanation for this is the ejection of a significant fraction of the baryons from the galaxy disc.

The observational evidence of galactic outflows is impossible to ignore (a review of both the theory and ob-

* Email: kellerbw 'at' mcmaster.ca servations of galactic winds can be found in Veilleux et al. 2005). From intergalactic metals seen in quasar absorption lines (Songaila \& Cowie 1996; Davé et al. 1998; Weiner et al. 2009), to the detection of neutral (Kunth et al. 1998; Morganti et al. 2003), ionized (Heckman et al. 1987; Martin et al. 2012), and molecular (Stark \& Carlson 1984) outflowing gas with outflow velocities $\gg 100 \mathrm{kms}^{-1}$ (Leroy et al. 2015), we can see that galaxies do not merely accrete gas: they eject it as well (a detailed study of gas inflows and outflows can be found in Woods et al. (2014)). In our own Milky Way, X-ray observations have detected $\sim 6 \times 10^{10} M_{\odot}$ in the $\sim 10^{6} \mathrm{~K}$ circum-galactic medium (CGM) (Gupta et al. 2012), comparable in mass to total baryonic mass of the galactic disc.

In Keller et al. (2015), we showed that a correct treatment of superbubbles driven by supernovae (SN) directly generates strong galactic winds without requiring extra wind models. This was the first time the complete physics of thermal conduction and evaporation required to model superbubbles (see Keller et al. 2014) was employed in galaxy formation simulations. This removes the need for cooling shutoffs, hydrodynamic decoupling, or other purely numerical boosts to the feedback effectiveness and the associated 
free parameters. These first principles outflows give a stellar mass evolution that matches the abundance matching results (Behroozi et al. 2013). By removing preferentially low-angular momentum gas, these outflows can prevent the growth of a large bulge, producing bulgeless discs like those that are seen in the nearby universe.

Prior work (Hopkins et al. 2014; Agertz \& Kravtsov 2015; Stinson et al. 2013) has examined the role of outflows in enabling galaxies to regulate their baryon content and match observed relations as a function of their mass. A common outcome is that stellar feedbacks are insufficient for higher mass galaxies. Typically, these works relied on multiple feedbacks and detailed subgrid models with associated free parameters. These additional feedbacks are discussed in more detail in section 2. They did not however, include necessary physics to accurately model superbubbles. Thus the question what supernovae driven outflows can do has yet to be fully answered. For example, Hopkins et al. (2014) has argued that supernovae alone cannot regulate the stellar content of intermediate mass galaxies, let alone the more massive ones.

The primary goal of this work is to examine galaxies and their outflows via a suite of simulated field $L *$ galaxies, the McMaster Unbiased Galaxy Simulations 2 (MUGS2). We begin with an overview of stellar feedback and galactic outflows in section 2. We examine which forms of feedback are most likely to contribution outflows and also discuss alternatives to supernovae. In section 3 , we describe the MUGS2 sample and simulation methods. We then examine the evolution and final state of the galaxy sample in section 4. As in other work, we find that galaxies more massive than $10^{12} \mathrm{M}_{\odot}$ deviate from observed relations. In our case, this is where supernova-driven winds begin to fail. We find a universal relation between the halo/disc mass and outflow mass loadings. Finally, in section 5, we discuss how stellar mass regulation through supernovae fails, how this manifests in the galaxy properties and how this provides strong clues that Active Galactic Nuclei (AGN) must take over the regulation.

\section{WHAT LAUNCHES GALACTIC OUTFLOWS?}

The question of what ultimately powers galactic winds has been debated for over half a century, since the first outflows were discovered in M82 (Lynds \& Sandage 1963). Unfortunately, neither (AGN) nor stellar processes have unambiguous observational signatures in the outflows they produce (Veilleux et al. 2005). To complicate matters further, stellar feedback comes in multiple forms. Disentangling these adds to the uncertainty of how galactic outflows are actually generated. Massive stars deposit energy and momentum in the ISM through ionizing radiation, radiation pressure on dust grains, stellar winds, and ultimately explode as supernova. Each of these processes, in principle, has sufficient energy to drive a galactic outflow. Radiation, in particular, has orders of magnitude more energy available than the others (Leitherer et al. 1999). Driving effective galactic winds requires strong coupling to the ISM gas and limited cooling losses so that the terminal velocies are high relative to the escape velocity. We examine the feedback processes individually below.

\subsection{Stellar Feedback}

\subsubsection{Ultraviolet Radiation \&5 HII Regions}

The characteristic temperature of gas photoionized by UV radiation is $\sim 10^{4} \mathrm{~K}$ Krumholz \& Matzner (2009), far lower than the virial temperature of all but the smallest galaxies. For galaxies with halo masses below $10^{9} \mathrm{M}_{\odot}$, photoheating and photoionization by UV radiation strong limits star formation (Efstathiou 1992). The characteristic sound speed for gas in HII regions is $\sim 10 \mathrm{~km} / \mathrm{s}$ is similar to characteristic turbulent velocities and escape speeds in molecular clouds. Even large HII regions, such as 30 Doradus, have been observed to have expansion rates of only $25 \mathrm{~km} / \mathrm{s}$ (Chu \& Kennicutt 1994), significantly less than what is needed to drive even a weak galactic fountain. Thus it is doubtful that UV radiation plays much role in launching galactic-scale outflows.

Simulations by Dale et al. (2012) have shown that HII regions alone are unable to effectively remove gas from their birth clouds, let alone their galaxies but can alter their structure. Thus UV can play a local role in simulations that resolve molecular clouds.

\subsubsection{Radiation Pressure}

Radiation pressure on the dust grains in galaxies is a potential driver for galactic outflows. Murray et al. (2011) showed that, assuming spherical symmetry, the most massive star clusters can drive reasonably fast ( $v \sim$ hundreds of $\mathrm{km} / \mathrm{s}$ ) outflows. Agertz \& Kravtsov (2015) and Hopkins et al. (2014) also found that a combination of SN, stellar winds, and radiation pressure produced a galaxy which matched the abundance-matched stellar mass fractions of Behroozi et al. (2013). However, as Agertz \& Kravtsov (2015) noted, and Roškar et al. (2014) examined in detail, these simulated galaxies often displayed unrealistic morphologies, with much thicker discs than would be expected for a normal MilkyWay like disc. Roškar et al. (2014) found the addition of radiation pressure using a local 'UV escape probability' model for dust absorption, with a single parameter for the IR dust opacity $\kappa_{I R}$ was able to reduce the stellar mass fraction of a cosmological galaxy with mass comparable to the lighter members of our well-regulated population. However, this required large values of $\kappa_{I R}$, such that the UV radiation coupled to the ISM so strongly that the entire disc was completely disrupted, and the resulting galaxy was completely spheroidal, with stellar scale heights above $3 \mathrm{kpc}$.

The porosity of the ISM can significantly reduce the mean optical depth of the galaxy, giving photons an escape valve to leave unimpeded (Krumholz \& Thompson 2013). The importance of radiation pressure remains an open question.

\subsubsection{Supernovae and Stellar Winds}

Based on energetics alone, it might seem that SN alone could eject gas from even the most massive halos. A Type II SN releases $\sim 10^{51} \mathrm{erg}$ in an ejecta of $\sim 10 \mathrm{M}_{\odot}$ (Leitherer et al. 
1999). This results in a maximum ejecta temperature of $\sim 2 \times 10^{8} \mathrm{~K}$. This corresponds to the virial temperature for halos with a mass of $\sim 10^{15} \mathrm{M}_{\odot}$. This fails to take into account the mixing of SN ejecta with the surrounding ISM. Additionally, if SN ejecta left a galaxy without any mixing or entrainment of additional gas, the largest wind mass loadings seen would be $\eta \sim 0.1$. Such winds would remove metals from the ISM but have little effect on the total baryon content. In order to moderate both star formation and bulge growth, mass loadings of $>10$ are necessary. Mixing in cooler ISM material reduces the effective wind temperature to $\sim 10^{6} \mathrm{~K}$ if cooling losses are small.

While it is well known that individual supernovae experience strong cooling, clustered star formation allows supernovae and stellar winds to combine into a superbubble that retains $65 \%$ of its initial injected energy even after the formation of cold shell which then breaks out of the ISM into the halo (Mac Low \& McCray 1988). With low density channels up to $99 \%$ of the energy can escape (Rogers \& Pittard 2013). An important function of the early stellar feedbacks discussed earlier is to clear dense gas around the star cluster to enable the superbubble to escape. However, as discussed previously, this is only valid in a simulation if dense gas is resolved. Thus superbubble feedback can be much more efficient that supernova feedback. The physics of evaporation also leads to specific predicitions with respect to mass loading and outflow temperatures of order $\sim 10^{6} \mathrm{~K}$ (Keller et al. 2014).

$\sim 10^{6} \mathrm{~K}$ is the virial temperature of halos with masses of a few $10^{12} \mathrm{M}_{\odot}$. If the wind fluid is cooler than the virial temperature, it will not be rise buoyantly out of the disc into the circum-galactic medium (CGM). This suggests SN driven winds become less effective, and may fail to launch altogether somewhere in the mass range of $\sim 10^{12} \mathrm{M}_{\odot}$. The existence of a peak in the star formation efficiency at this same mass, as seen in Behroozi et al. (2013) is strong evidence that this indeed be occurring in nature, and that some other feedback mechanism begins to dominate at higher masses. Finding out the if, when and how of this transition is important if we want to know how larger galaxies quench their star formation, and if SN alone can explain the low star formation efficiency in galaxies below this mass.

\subsection{Other Feedback Mechanisms}

\subsubsection{AGN}

The primary non-stellar energy source available for driving galactic outflows is feedback from the growth of supermassive black holes (SMBHs). The Milky Way's own SMBH has a mass of $M_{\bullet} \sim 4 \times 10^{6} \mathrm{M}_{\odot}$ (Meyer et al. 2012). The energy released by it's formation is $M_{\bullet} c^{2} \sim 7 \times 10^{60} \mathrm{erg}$. This is significantly greater than the binding energy of the galactic halo $\left(\sim 10^{59} \mathrm{erg}\right)$. If even a small fraction of this energy couples to the ISM as it is released, it can have a significant disruptive effect. SMBHs are ubiquitous and larger ones are strongly linked to the presence of massive bulges (Magorrian et al. 1998).

Much effort has gone into developing sub-grid models for AGN feedback and SMBH growth, and now AGN feedback is a major component of many large box simulations (e.g., Illustris (Sijacki et al. 2015), EA-
GLE (Crain et al. 2015), Rhapsody-G (Hahn et al. 2015), Horizon-AGN (Dubois et al. 2014))

\subsubsection{Cosmic Rays}

Cosmic rays (CRs) have been been proposed as another potential engine for driving outflows (Ipavich 1975; Breitschwerdt et al. 1991; Everett et al. 2008; Socrates et al. 2008). Cosmic rays could be directly linked to SN shocks or shocks within the ISM. Cosmic rays may contain as much energy as the thermal and magnetic components of a galaxy (Zweibel \& McKee 1995).

Jubelgas et al. (2008) found CR had little impact on higher mass galaxies. Girichidis et al. (2015) showed that cosmic rays can launch winds with mass loadings of order unity for gas surface densities comparable to the Milky Way. Salem \& Bryan (2014) and Booth et al. (2013) found CR driven outflows had even lower mass loadings for Milky Way mass halos. These results make it doubtful that cosmic rays are centrally important for galactic outflows.

\section{METHODS: MUGS2}

The simulations presented here are the new MUGS2 simulations. The original MUGS sample, presented in (Stinson et al. 2010), followed the evolution of isolated, Milky-Way like disc galaxies using low temperature metal cooling, UV background radiation, and stellar feedback. The new MUGS2 sample includes all of this, plus a number of improvements to the hydrodynamic method, along with the new superbubble feedback model (Keller et al. 2014). This has resulted in significantly different evolution in the MUGS2 sample compared to the original MUGS set, most notably greatly reduced star formation in nearly every galaxy (the original MUGS sample greatly overproduced stars).

These simulations were run using the modern smoothed particle hydrodynamics (SPH) code GASOLINE2, as in Keller et al. (2015) and Keller et al. (2014). The changes in this new code include a sub-grid model for turbulent mixing of metals and energy (Shen et al. 2010), and a modified pressure force form similar to that proposed by Ritchie \& Thomas (2001), which is functionally equivalent to Hopkins (2013). Details of the star formation and gas physics model can be found in Keller et al. (2014).

\subsection{Simulation Initial Conditions}

We adopt the same initial conditions (ICs) that were used in the original Stinson et al. (2010) MUGS sample. These cosmological zoom-in ICs were selected from a $50 h^{-1} \mathrm{Mpc}$ cube, run (with dark matter only) to $z=0$. The simulations use a WMAP $3 \Lambda$ CDM cosmology, with $H_{0}=73 \mathrm{~km} / \mathrm{s} / \mathrm{Mpc}$, $\Omega_{M}=0.24, \Omega_{\text {bary }}=0.04, \Omega_{\Lambda}=0.76$, and $\sigma_{8}=0.76$. Galaxies that had halo mass from $5 \times 10^{11} \mathrm{M}_{\odot}$ to $2 \times 10^{12} \mathrm{M}_{\odot}$ were then selected from the dark matter only run. Isolated galaxies were then selected by choosing only ones which had no neighbours in this mass range within 2.7 Mpc. This resulted in a sample of 276 candidate halos, of which the final MUGS sample was simply selected at random from this pool. While 
only 9 of these were presented in Stinson et al. (2010), subsequent papers presented the remaining galaxies (Bailin et al. 2010; Nickerson et al. 2011, 2013), and what we present here is the full set of galaxies that were produced for MUGS.

Each of these simulations has a gas mass resolution of $M_{\text {gas }}=2.2 \times 10^{5} \mathrm{M}_{\odot}$, and uses a gravitational softening length of $\epsilon=312.5 \mathrm{pc}$, and a minimum SPH smoothing length set to $1 / 4$ of this. The total sample presented here consists 18 galaxies, with $\mathrm{z}=0$ virial masses in a range from $3.7 \times 10^{11} \mathrm{M}_{\odot}$ to $2.1 \times 10^{12} \mathrm{M}_{\odot}$.

\subsection{Star Formation}

We use a standard Schmidt-law star formation recipe, where the rate is set by the freefall time of gas and an efficiency $c_{*}:$

$\dot{\rho}_{*}=\frac{c_{*} \rho}{t_{f f}}=c_{*} \sqrt{\frac{32 G}{3 \pi}} \rho^{3 / 2}$

We used an efficiency of $c_{*}=0.05$, as was used for the original MUGS simulations (Stinson et al. 2010) and in Keller et al. (2015). Star formation is only allowed to take place in gas which satisfies three criteria: it has a temperature below $1.5 \times 10^{4} \mathrm{~K}$, density above $9.6 \mathrm{~cm}^{-3}$ (the density where gravitational softening becomes important), and is in a converging flow $(\nabla \cdot \vec{v}<0)$. This density threshold is larger than the that used in Stinson et al. (2010) (which used $0.1 \mathrm{~cm}^{-3}$, but identical to that used in Stinson et al. (2013). We have used this larger threshold (as Governato et al. (2010) recommends) to better capture the effects of clustered star formation. Aside from this higher star formation threshold, our star formation recipe remains unchanged from the original MUGS sample.

\subsection{Superbubble Feedback}

We use the superbubble feedback model described in Keller et al. (2014). We briefly summarize the important details below. The model deposits thermal energy and mass into resolution elements in a brief multi-phase state. These particles each have separate specific energies and masses for the hot bubble and cold ISM components, including the swept up shell. This allows the method to calculate a separate density, temperature, and cooling rate for each phase, rather than using the average density and temperature of both phases. Multiphase particles are also prevented from forming stars if the average temperature of the two phases is above our temperature threshold for star formation. The amount of supernova feedback per unit stellar mass is determined using a Chabrier (2003) IMF.

In Keller et al. (2014), we demonstrated that superbubble feedback could produce significantly more mass-loaded outflows and reduced star formation in simulations of isolated disc galaxies, compared to the older (Stinson et al. 2006) blastwave model. We also showed how the ISM phase behaviour in superbubble-regulated galaxy behaved in a much more physically reasonable way than in a galaxy regulated with single phases. Keller et al. (2015) showed that in a cosmological galaxy (one of the same galaxies presented here in the MUGS2 sample, g1536) superbubble feedback resulted in high mass loadings $\eta \sim 10$ for galactic outflows from $z \sim 4-2$, which then fell to $\eta \sim 1$ at low redshift, producing a galaxy with a realistic star formation history and no significant bulge component, without the need for additional feedback mechanisms, or greater feedback energies than what are provided for simply by SN alone. We also showed that this comes without additional computational cost, or the addition of any new free parameters/tuning.

With $\sim 10^{51} \mathrm{erg}$ coupled to the ISM per supernova this gives $\sim 10^{49} \mathrm{erg}_{\odot}^{-1}$ for the full stellar population. This is significantly less energy per mass of stars formed than models such as the FIRE (Hopkins et al. 2014) model, which included feedback from stellar winds and radiation pressure, or the large volume simulations like EAGLE (Schaye et al. 2015) or Illustris (Sijacki et al. 2015), which required artificially boosted feedback energies to match observed galaxy populations.

Aside from a different star formation density and temperature threshold, the star formation and feedback recipes used here identical to those used in Keller et al. (2014), and are completely identical to those used in Keller et al. (2015).

\section{RESULTS}

\subsection{Redshift Zero Properties}

The general properties of each galaxy at $\mathrm{z}=0$ can be found in table 1. $\lambda^{\prime}$ is the dimensionless spin parameter of the halo, defined by Bullock et al. (2001) as $\lambda^{\prime}=J / \sqrt{2 G M_{v i r}^{3} R_{v i r}}$. $f_{b}$ is the baryon fraction of the halo. $z_{1 / 2}$ and $z_{l m m}$ are the redshifts at which the halo reaches half of its final mass, and the redshift of last major merger respectively. Major mergers are defined to match Stinson et al. (2010), as a merger with a halo containing at least $1 / 3$ the stellar mass of the main progenitor. $M_{v i r}, M_{*}$, and $M_{\text {gas }}$ are the masses of the full halo, the stellar component, and the gas component respectively within the virial radius. $\mathrm{SFR}_{\mathrm{z}=0}$ is the redshift 0 star formation rate (SFR), averaged over the previous 100Myr. The virial radius is defined as the radius around the halo such that the enclosed density is 200 times the critical density $\left(\rho=200 \rho_{\text {crit }}\right)$. While we used the same definitions for quantities reported in Stinson et al. (2010), every value reported here is derived from the new MUGS2 simulations. We also define a central region of the halo, which contains the disc and the majority of the stars within the halo. The baryon mass within this region is given as $M_{\text {central }}$ This central region is simply a sphere of radius $0.1 R_{\text {vir }}$.

Mock stellar observations and HI column images of these galaxies can be seen in figures 1 and 2 . The varied merger history of these galaxies is evident in these images: companions can be seen in 3 galaxies (g21647, g22795, $\mathrm{g} 4145)$, tidal tails are evident in another (g19195), and strong bars exist in another 2 (g28547, g24334). The HI column density shown in figure 2 shows, as was seen in Keller et al. (2015), large quantities of extraplanar HI gas, driven up by outflows from the galaxy disc. The labels on each of these images are coloured red if they are in the "unregulated" population discussed in the next section. 


\begin{tabular}{rrrrrrrrrr}
\hline $\begin{array}{r}\text { Galaxy } \\
\text { ID }\end{array}$ & $M_{\text {vir }}$ & $\lambda^{\prime}$ & $f_{b}$ & $z_{1 / 2}$ & $z_{l m m}$ & $\begin{array}{r}M_{*} \\
\text { (masses }\end{array}$ & $\begin{array}{r}M_{\text {gas }} \\
\text { in }\end{array}$ & $\begin{array}{r}M_{\text {central }} \\
\left.10^{10} M_{\odot}\right)\end{array}$ & $\begin{array}{r}S F R_{z=0} \\
\left(M_{\odot} y r^{-1}\right)\end{array}$ \\
\hline \hline g7124 & 36.6 & 0.039 & 0.150 & 0.8 & 2.1 & 0.5 & 5.0 & 1.8 & 0.6 \\
g5664 & 47.7 & 0.028 & 0.173 & 0.8 & 3.0 & 0.9 & 7.3 & 3.0 & 1.7 \\
g8893 & 58.0 & 0.065 & 0.170 & 1.0 & 0.8 & 0.7 & 9.1 & 2.6 & 1.0 \\
g1536 & 64.9 & 0.029 & 0.189 & 1.0 & 4.0 & 1.9 & 10.4 & 5.2 & 3.9 \\
g21647 & 74.4 & 0.069 & 0.152 & 0.2 & 0.5 & 1.2 & 10.1 & 3.1 & 2.7 \\
g422 & 76.2 & 0.033 & 0.183 & 0.8 & 0.7 & 1.5 & 12.4 & 5.1 & 3.2 \\
g22437 & 85.2 & 0.013 & 0.192 & 0.7 & 2.6 & 9.0 & 7.3 & 11.2 & 17.9 \\
g22795 & 85.2 & 0.009 & 0.178 & 1.1 & 3.8 & 10.6 & 4.6 & 11.7 & 6.2 \\
g3021 & 97.8 & 0.040 & 0.192 & 0.6 & 1.7 & 3.6 & 15.1 & 9.9 & 15.7 \\
g28547 & 98.5 & 0.106 & 0.186 & 0.4 & 0.1 & 1.6 & 16.7 & 4.5 & 2.7 \\
g19195 & 101.6 & 0.039 & 0.162 & 0.7 & 0.7 & 7.1 & 9.3 & 9.7 & 20.3 \\
g24334 & 102.2 & 0.052 & 0.174 & 0.5 & 1.6 & 2.6 & 15.3 & 7.2 & 6.8 \\
g4720 & 102.5 & 0.015 & 0.192 & 0.8 & 1.8 & 14.2 & 5.5 & 14.4 & 8.3 \\
g4145 & 119.5 & 0.033 & 0.193 & 0.9 & 1.4 & 15.0 & 8.1 & 16.9 & 20.9 \\
g25271 & 125.5 & 0.016 & 0.187 & 1.1 & 4.0 & 15.6 & 7.9 & 17.0 & 9.7 \\
g15784 & 131.2 & 0.037 & 0.186 & 1.3 & 7.3 & 13.0 & 11.4 & 17.7 & 9.6 \\
g15807 & 203.2 & 0.026 & 0.191 & 1.0 & 2.5 & 21.4 & 17.5 & 25.4 & 11.9 \\
g27491 & 214.7 & 0.039 & 0.184 & 0.7 & 1.1 & 18.8 & 20.8 & 26.6 & 17.6 \\
\hline
\end{tabular}

Table 1. Redshift 0 properties of the full MUGS2 sample. All masses and particle counts are measured within a $R_{v i r}$ sphere centered on the halo, except for $M_{\text {central }}$, which is measured within a $0.1 R_{v i r}$ sphere.
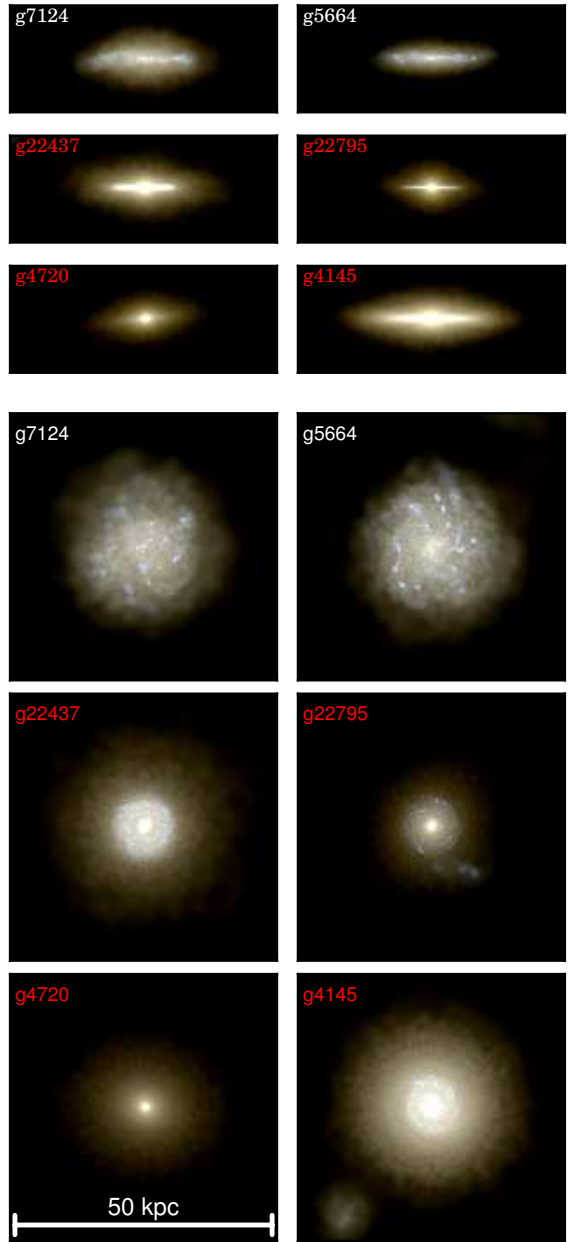
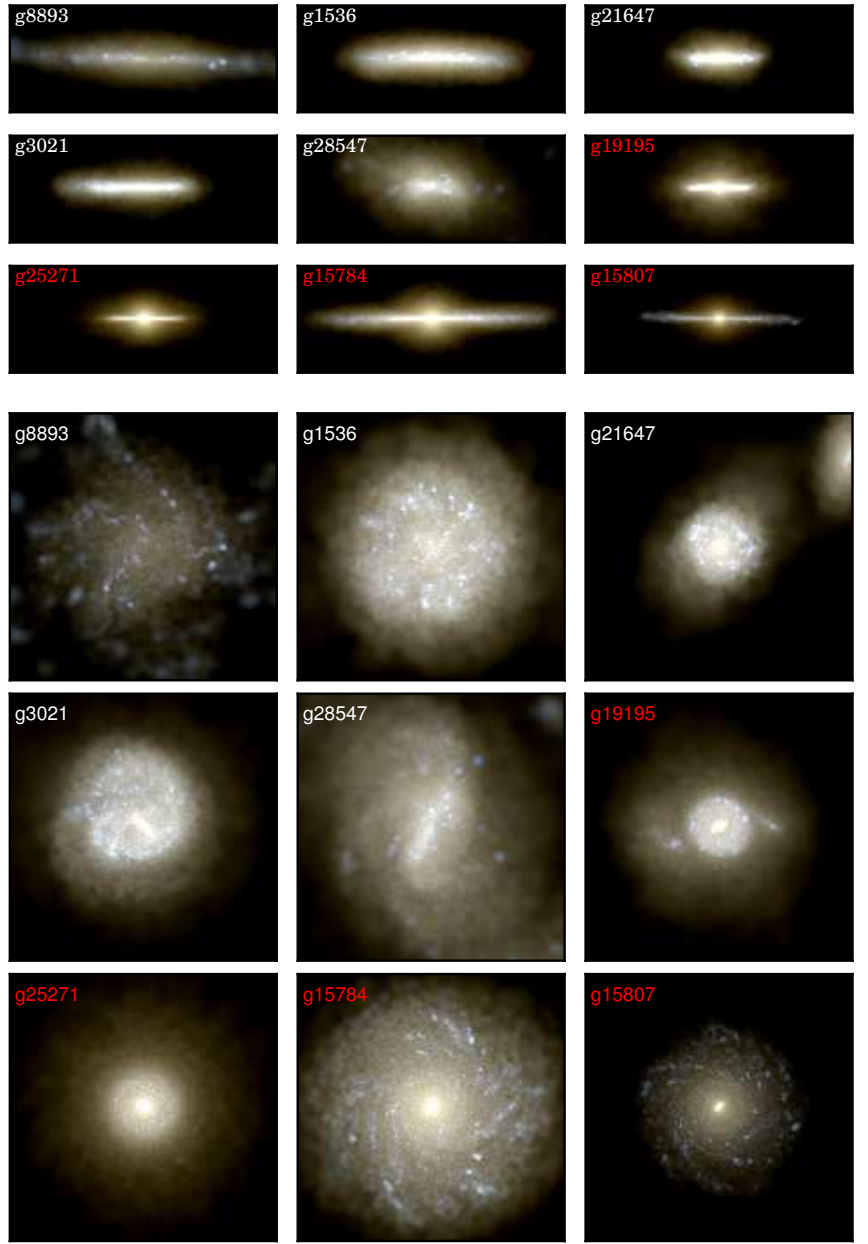

Figure 1. Mock optical stellar observations of each MUGS2 galaxy. RGB channels are calculated using Marigo et al. (2008) stellar populations. The top 3 rows show the galaxies edge on, while the bottom 3 show the galaxies face on. The galaxies are sorted in order of halo mass, and galaxies that overproduce stars are labelled with red. These images do not include the effects of dust extinction 

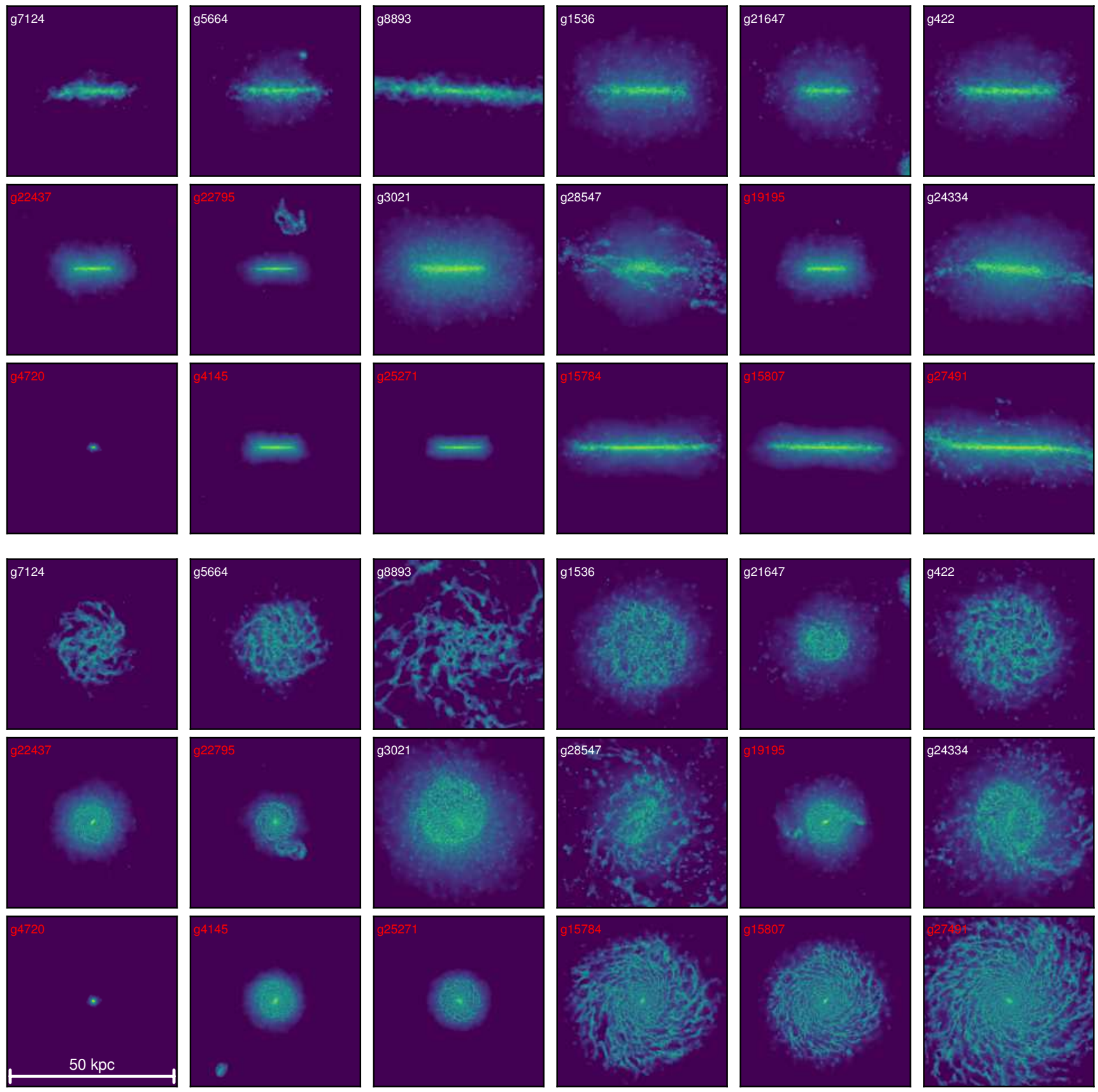

Figure 2. HI column density in each of the MUGS2 galaxies. As in figure 1, the top 3 rows show the galaxy edge on.

\subsection{Stellar Mass Runaway}

As can be seen in figure 3, roughly half of these galaxies fall within the expected $z=0$ stellar mass to halo mass relation (SMHMR). The grey band shown in the figure is the $2 \sigma$ confidence region in the observed SMHMR from Hudson et al. (2015), which used the CFHTLens weak lensing survey to produce a fully-observational SMHMR, without the uncertainties of the using dark matter-only simulations that are needed for abundance matching techniques (the Hudson et al. (2015) SMHMR is consistent with past abundance matching estimates such as Behroozi et al. (2013) and Moster et al. (2013)). For the rest of this paper, we will refer to the galaxies that fall within the $2 \sigma$ confidence interval for the observed SMHMR (i.e. galaxies where stellar feedback prevents overproduction of stars) as well-regulated, and the galaxies where stellar feedback fails to produce the correct SMHMR as unregulated. No other criterion is used for determining which population a galaxy falls into. In addition to overproducing stars, these unregulated galaxies are qualitatively redder and more bulge dominated, as can be seen in the mock stellar images of figure 1. There appears to be no significant difference in terms of merger activity between the two populations, strongly suggesting that the failure of stellar feedback to regulate the galaxies is not simply a matter of recent or violent merging. The rotation curves in figure 4 


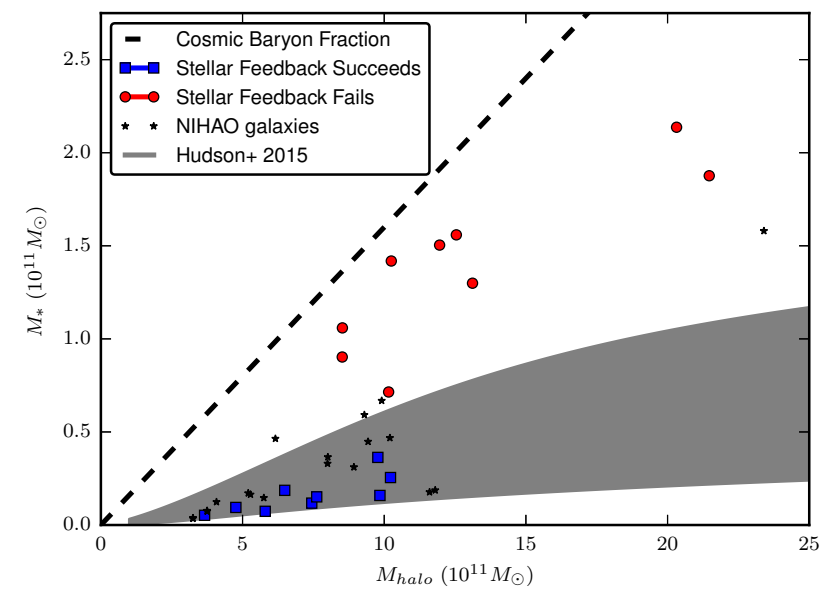

Figure 3. Stellar mass vs. halo mass at $\mathrm{z}=0$. The observed stellar mass to halo mass relation is from Hudson et al. (2015)'s weak lensing study of galaxies to $\mathrm{z}=0.8$. Galaxies that fall within this range are shown as blue squares, while galaxies above the curve (those which form too many stars) are shown as red circles. For comparison, the black stars show galaxies from the NIHAO sample (Wang et al. 2015).

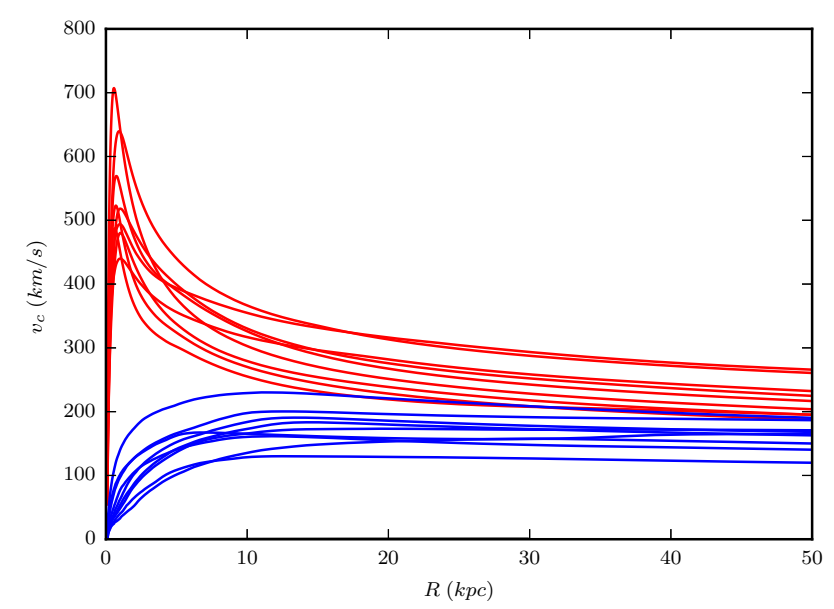

Figure 4. As is clear from the above rotation curves, galaxies which overproduce stars (shown in red) also have large central concentrations, giving steeply peaked rotation curves inconsistent with those seen in local $L *$ galaxies. Galaxies with well-regulated star formation have flat rotation curves (shown in blue).

also show the distinct signature of massive bulges formed by catastrophic angular momentum loss in the unregulated population. van den Bosch (2001) showed that this can arise when gas simply traces the dark matter distribution, without redistribution or ejection by feedback. The strong peaks (as high as $700 \mathrm{~km} / \mathrm{s}$ for g4720) come about from the central concentration of baryons in the galaxy bulge. Without exception, each of the well-regulated galaxies shows a flat rotation curve, with no evidence of a significant bulge component. This matches the qualitative morphology seen in figure 1.

Figure 5 shows that for the well-regulated population, the stellar mass and central baryonic mass follow an extremely tight linear relation, with a mean star formation effi-

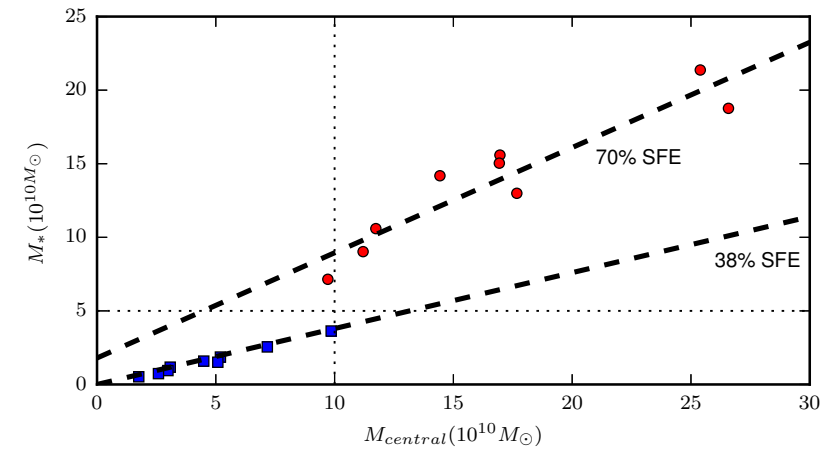

Figure 5. The stellar mass and the central baryonic mass show a tight correlation. For the well-regulated population, roughly $40 \%$ of the central baryons are stars, while for the unregulated population has converted $\sim 70 \%$ of the central baryon mass into stars.

ciency (simply defined here as the fraction of central baryons that are in stars) of $38 \pm 2 \%$ over a Hubble time. For the galaxies that fail to self-regulate, they exhibit a total star formation efficiency of $70 \pm 10 \%$, converting the majority of the baryons that collapse onto their disc into stars. Interestingly, the two populations can be divided cleanly along the $M_{\text {central }}=10^{11} \mathrm{M}_{\odot}$ or the $M_{*}=5 \times 10^{10} \mathrm{M}_{\odot}$ axis. This is clear evidence that what explains the dichotomy here must involve the accretion (or the failure to remove!) baryons from the central region of the halo, where the disc resides and stars are formed.

The gas phase diagram of galaxies in the two populations is remarkably similar, as can be seen in figure 6 . For a representative pair from each population, of nearly equal halo mass, gas follows essentially the same evolutionary path, characteristic of a superbubble-regulated ISM, as was shown in Keller et al. (2015). Gas accretes from the halo, building up a warm ISM at $T \sim 10^{4} \mathrm{~K}$. Where that gas reaches densities of $\sim 1 \mathrm{~cm}^{-3}$, it begins to cool quickly and form stars. Those stars begin to explode as SN, and a hot superbubble is formed, with $T>10^{7} \mathrm{~K}$. As the bubble grows, it's temperature falls as it both expands adiabatically and evaporates the cold shell surrounding it. This hot, buoyant gas leaves the disc, rising through the CGM and cooling adiabatically as it goes. What is remarkable here is just how similar the phase diagrams of these two galaxies are. The slight differences are what we would expect from table 1 . g19195 has 50\% less gas than g24334, and more than 3 times the SFR at $z=0$. Thus, there is less total material, especially in the warm \& cold phases, and slightly more of the very hot $\left(T>5 \times 10^{7} \mathrm{~K}\right)$ gas (the interiors of very young superbubbles) as a consequence of the higher SFR. What is important to take away from these phase diagrams is that a well-regulated and unregulated galaxy do not have any real difference in the phase behaviour of their gas. There is no runaway cooling, or failure of SN to generate hot gas: what causes the unregulated population to runaway is purely an effect of the outflow effectiveness, which is set by the depth of the potential well. 

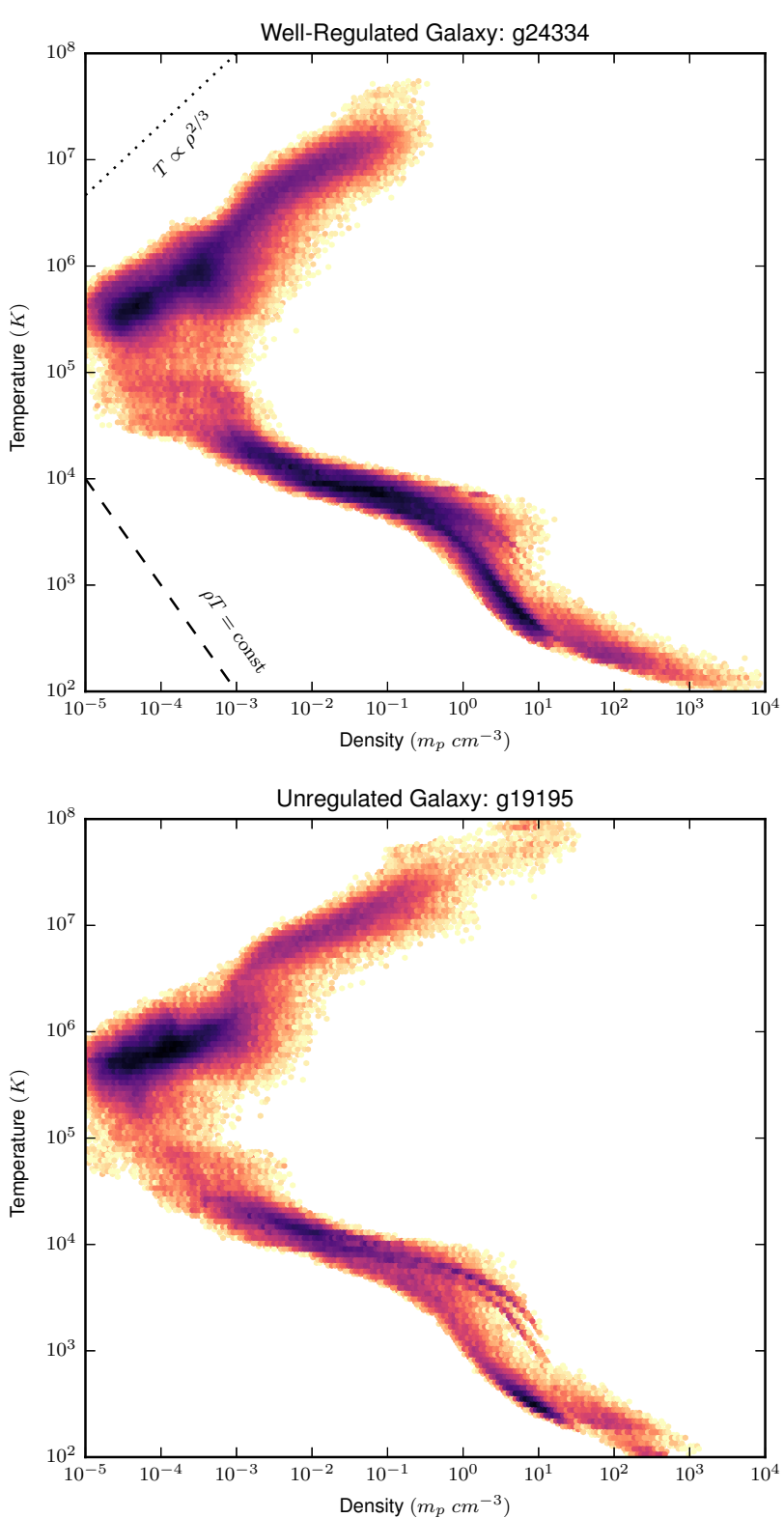

Figure 6. Gas phase diagrams for a well-regulated galaxy (g24334) and an unregulated galaxy (g19195). Color shows the amount of mass at a given $\rho-T$ point. The top phase diagram shows the characteristic slope for adiabatic (dashed line) and isobaric (dotted line) processes. As can be seen, both galaxies are qualitatively the same. Both show the equilibrium cooling curve below $10^{4} \mathrm{~K}$, and both show the adiabatic evolution of superbubble-heated gas as it leaves the disc.

\subsection{Time Evolution}

The obvious question that arises from the presence of these two (well-regulated vs. unregulated) populations at $z=0$ is whether they are distinct through their entire evolution, and if not, why/how do they diverge? Previous work suggests that a characteristic (halo or stellar) mass exists above which AGN feedback is needed. There is much uncertainty, however, about where this mass exactly is, and how the transition from SN to AGN regulation actually works. We should thus expect galaxies to begin diverging once this character-

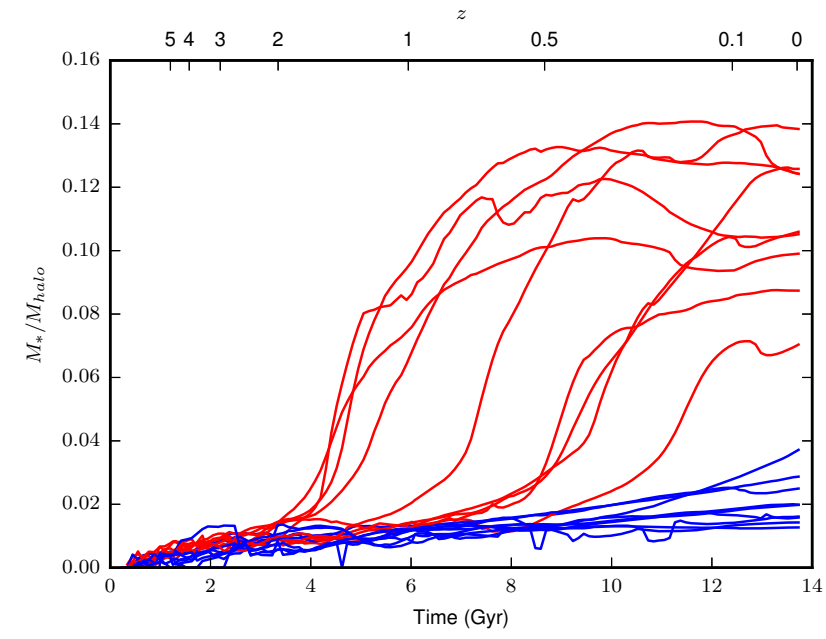

Figure 7. Stellar mass fraction vs. time. As is clear, for the unregulated population the buildup of stellar mass above $\sim 3 \%$ of the halo mass happens very rapidly, often on timescales of $\sim 1 \mathrm{Gyr}$. The runaway in the unregulated population stops simply when the disc has been sufficiently depleted of gas that little remains to form new stars.

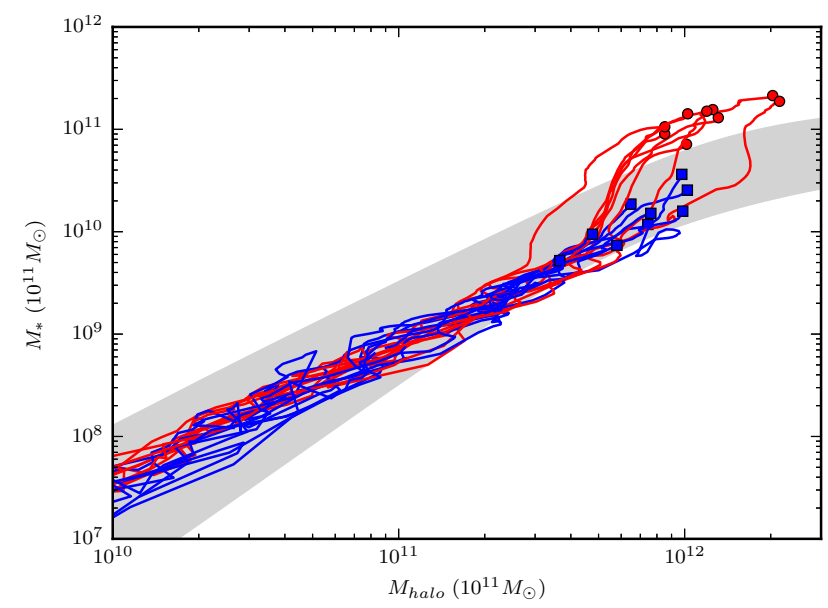

Figure 8. Stellar mass vs. halo mass, as shown in figure 3, but with trails added to show time evolution. For halo masses below $\sim$ a few $\times 10^{11}$, the evolution of the regulated and unregulated populations are indistinguishable. The grey bar shows the $z=0$ SMHMR, as in figure 3

istic mass is exceeded. As figure 7 shows, the stellar mass fraction for the two populations appear to follow similar evolutionary tracks for some time (until past $z=0.5$ in the case of g19195, the galaxy which diverges the latest). However, once a galaxy begins to overproduce stars, it appears to do so in a runaway, doubling or even tripling its stellar mass fraction in less than a Gyr.

The similar early star formation history of the two populations is even more clear if we look at the SMHMR's time evolution in figure 8 . Here we can see, that for galaxies with halo masses below a few $10^{11} \mathrm{M}_{\odot}$, the stellar mass falls well within the expected SMHMR distribution, and the two populations are indistinguishable. The mass at which the populations begin to diverge $\left(M_{*} \sim 10^{10} \mathrm{M}_{\odot}\right.$ and 


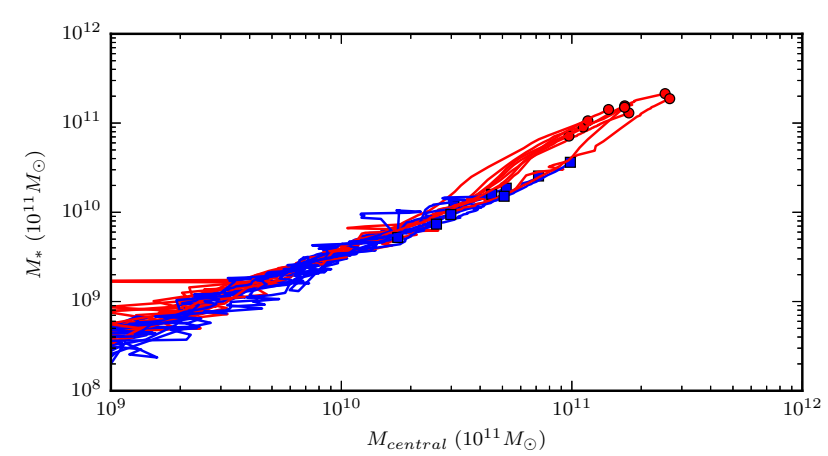

Figure 9. Stellar mass vs. central baryon mass. The stellar mass here can be seen to vary much more smoothly over the evolution, without the slight "jump" seen in figure 7 .

$\left.M_{v i r} \sim 3-5 \times 10^{11} \mathrm{M}_{\odot}\right)$ are quite close to the expected transition range from Shankar et al. (2006) $\left(M_{*} \sim 1.2 \times 10^{10} \mathrm{M}_{\odot}\right.$ and $\left.M_{\text {vir }} \sim 3 \times 10^{11} \mathrm{M}_{\odot}\right)$.

The relatively smooth change in the $M_{*}-M_{\text {central }}$ relation, shown in figure 9, shows that it is in fact the central baryon mass that is more tightly correlated with the stellar mass than the total halo mass (in galaxies regulated by SN feedback alone). In fact, it is likely that some of the more massive galaxies in the well-regulated sample are on their way to failing, but simply have not yet had enough time by $z=0$ to diverge significantly from the observed SMHMR. Once more than $\sim 10^{11} \mathrm{M}_{\odot}$ of baryons have accreted to the disc of a galaxy, SN alone appear to be unable to halt the growth of stellar mass. At this point, star formation efficiency of the central disc begins to increase, from $\sim 40 \%$ to $\sim 70 \%$, resulting in the differences seen in the two populations at $z=0$. This accretion crisis, as it continues to smaller scales, also explains the rotation profiles of the unregulated population. The overcollapse that results in runaway starformation also builds a massive bulge, producing the peaked rotation curves seen in figure 4 .

\subsection{Galactic Outflows}

A key metric for both observational and simulation studies of galactic outflows is the mass loading of these outflows. This scaling is usually written as a power-law scaling between mass loading $\eta$ and the circular velocity of the halo or halo mass (Murray et al. 2005; Peeples \& Shankar 2011):

$\eta \propto v_{c}^{-\alpha}=\left(G M_{\text {halo }} / R_{\text {vir }}\right)^{-\alpha / 2} \propto M_{\text {halo }}^{-\alpha / 3}$

The normalization of this relation characterizes how generally effective stellar feedback is at driving galactic winds and outflows. The index $\alpha$ is a measure of how this effectiveness decreases for larger halos, and ultimately results in a shutdown of large-scale outflows at high enough mass. This makes this scaling relation a key parameter to most semi-analytic galaxy formation models (e.g Cole et al. (2000)), and an attractive target for both theorists and observers alike. Two primary modes for driving galactic winds have been proposed. Energy-driven winds (such as those investigated by Mac Low \& McCray (1988); Tegmark et al. (1993), etc.) assume the cooling times for outflowing material are shorter than the time required for a superbubble

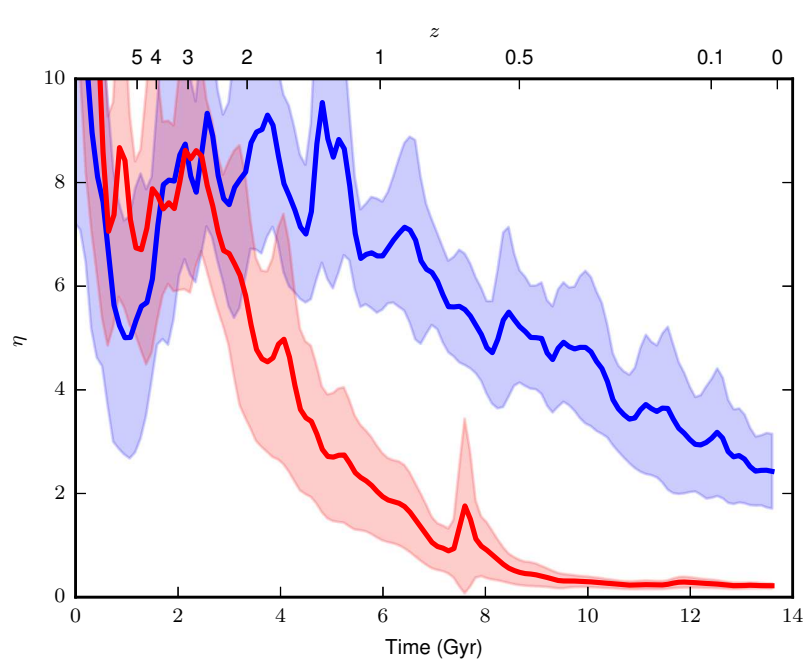

Figure 10. Outflow mass loading decreases as galaxies grow over time, as was previously shown by Keller et al. (2015). Here we see, when examining the mean mass loadings for the two populations (the well-regulated galaxies, where stellar feedback succeeds, and the unregulated galaxies, where it fails), that the unregulated galaxies have their mass loadings decrease sooner, and to lower values, than the well-regulated population. The error bars here are the $1 \sigma$ scatter in each population.

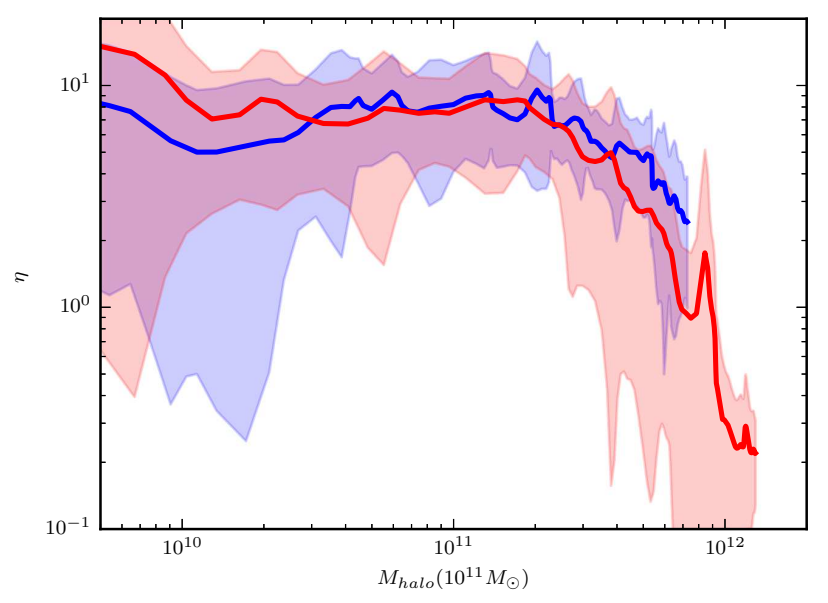

Figure 11. Unlike in the previous figure, if we look at mass loading as a function of halo mass, we instead see no significant difference between the two populations. Instead, both follow a similar trajectory, and the halos which fail to self-regulate do so simply because they reach a higher mass earlier.

to break out of the galactic disc. These adiabatically expanding bubbles result in galactic winds with mass loadings that scale as $\eta \propto v_{c}^{-2}$. A recent study by Christensen et al. (2015) showed that SN driven winds follow this scaling, using mock observations of a sample of 7 simulated galaxies. If the cooling times are instead much shorter than the break out time, then outflows become momentum driven (Murray et al. 2005), and the mass loadings instead are expected to scale as $\eta \propto v_{c}^{-1}$. Peeples \& Shankar (2011) used a semi-empirical model to fit the mass-metallicity relationship in low redshift galaxies to predict that the mass loading 
index must be $\alpha=3$ or steeper in order to produce a massmetallicity relation as steep as is observed.

As in Keller et al. (2015), we define our outflow rate using the simple formula below, for gas particles that have $v_{r}>0$, and are found between $0.1 R_{v i r}$ and $R_{v i r}$ :

$\dot{M}_{\text {out }}=\sum_{r_{i} \in \text { shell }} \frac{M_{i} \vec{v}_{i} \cdot \hat{r}}{0.9 R_{\text {vir }}}$

This choice reflects the nature of these outflows, where gas at high temperatures and low densities drifts outwards from the galaxy towards the virial radius. We chose a relatively thick shell to avoid the need for frequent simulation outputs. As such, our ability to resolve short-term bursts is somewhat reduced, but the overall outflow rates trace those from a test case with $8 \times$ more frequent outputs, and a shell $8 \times$ thinner. An excellent discussion of how to calculate outflow rates can be found in Appendix A of Muratov et al. (2015).

If we take the mean mass loadings $\eta=\dot{M}_{\text {out }} /$ SFR of our two populations, we see in figure 10 that the mass loading for the unregulated population drops below $\eta=1$ at $z \sim 0.5$, while the well-regulated population never drops below $\eta \sim$ 2. This helps explain the correlation between the central concentration and stellar mass fraction (in particular, the dichotomy between the two populations, with low central baryon masses for well-regulated galaxies and high central masses for the unregulated galaxies). Failure to eject gas through outflows gives a higher central baryon mass, and those baryons inevitably become stars. However, we know that the unregulated population is on average heavier (both the full halo, as well as the central baryons). Does this earlier drop in outflow mass loading come about simply because the unregulated population gets heavier earlier?

Figure 11 suggests exactly that. The mass loadings appear roughly constant, at $\eta \sim 10$ for most of the mass-range for both populations, but begin to fall at essentially the same mass, at what appears to be the same rate. An even tighter relation is seen in figure 12 , when the relation between the central baryon mass, rather than just the halo mass, is examined. It appears that for the $\eta-M_{\text {halo }}$ and $\eta-M_{\text {central }}$ relation, a broken power-law fit, as defined below, describes both the regulated and unregulated population.

$\eta= \begin{cases}\alpha M^{\beta} & \text { if } M>M_{0} \\ \left(\alpha M_{0}^{\beta-\gamma}\right) M^{\gamma} & \text { if } M<M_{0}\end{cases}$

Using a simple non-linear least squares fit in the on the parameters $\alpha, \beta, \gamma$, and $M_{0}$, we find that the most likely value for these parameters are $\alpha=0.9 \pm 0.5, \beta=-0.01 \pm$ $0.06, \gamma=-1.3 \pm 0.1, M_{0}=10^{10.0 \pm 0.1}$ for the $\eta-M_{\text {central }}$ relation, and $\alpha=1 \pm 1, \beta=0.0 \pm 0.1, \gamma=-1.8 \pm 0.2$, $M_{0}=10^{11.37 \pm 0.08}$ for the $\eta-M_{\text {halo }}$ relation. A fit for both of the populations independently is consistent with these values derived for the full set of MUGS2 galaxies. Interestingly, neither of these slopes is consistent with simple energy or momentum driven winds (although a single power-law fit does give a slope of $\sim 0.5 \pm 0.2$, consistent with an energydriven scenario, albeit with a much weaker fit). These values are consistent, however, with the constraints derived for the mass-metallicity relation and gas content in nearby galaxies derived by Peeples \& Shankar (2011) (namely, that $\alpha$ in the $\eta \propto v_{c}^{-\alpha}$ relation be steeper than 3$)$.

The temperature of the outflows also shows an interesting trend as a function of halo mass. Figure 13 shows that

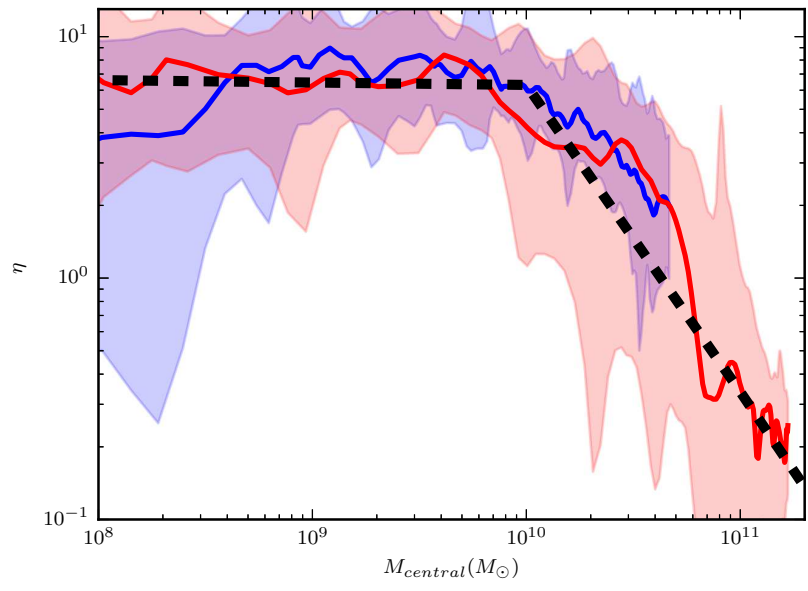

Figure 12. The mass loadings of the two populations are even more in agreement if we look at their relation to the central baryonic mass. As with the mass loading vs. halo mass relation, we can fit a broken power law to this relation (shown here as the dashed line).

as halo mass increases, the mass loading of outflows with cold gas $\left(T<10^{5} \mathrm{~K}\right.$. We choose this as a cutoff point as it lies near the peak of the cooling curve, where we would expect, as Woods et al. (2014) showed, very little gas to be found) monotonically decreases, while the loading of hot gas is convex, peaking at $M_{\text {halo }}=2-5 \times 10^{11} \mathrm{M}_{\odot}$, and then rapidly falling as halo mass increases. If we think in terms of virial temperature, this makes sense. At low $T_{v i r}$, gas around $10^{5} \mathrm{~K}$ is buoyant, and will be driven out of the disc. The halo itself is smaller, with a shallower potential well, thus making it easier for the same amount of feedback energy to eject a larger amount of entrained gas. As the halo mass grows, eventually only the hottest gas is able to escape, and only if it isn't weighed down by too much entrained material. Eventually, this effect means that the total mass loading begins to drop significantly.

We can see clearly in figure 14 that our winds essentially have a maximum effective velocity of $\sim 250 \mathrm{~km} / \mathrm{s}$. When the escape velocity at the edge of the galaxy (where we begin to measure outflows, at $0.1 R_{\text {vir }}$ ) exceeds this velocity, outflow mass loadings precipitously drop from $\sim 10$ to $<1$. As Keller et al. (2014) showed, superbubble-heated gas tends to reach an equilibrium temperature of a a few million K, meaning that the outflow rates appear to fall significantly when outflowing gas, moving faster than the escape velocity from the disc is moving supersonically relative to the superbubble-heated gas. Radiative losses from cooling shocks may be important here for causing the drop in outflow efficiency that produces our two populations.

\section{DISCUSSION}

In Keller et al. (2015), we showed that galactic outflows, driven by pure SN feedback modeled using the superbubble method of Keller et al. (2014) can produce a moderate mass $L *$ galaxy with a realistic star formation history and no significant bulge component. We have extended this work to a sample of 18 galaxies, covering a mass range of roughly an 


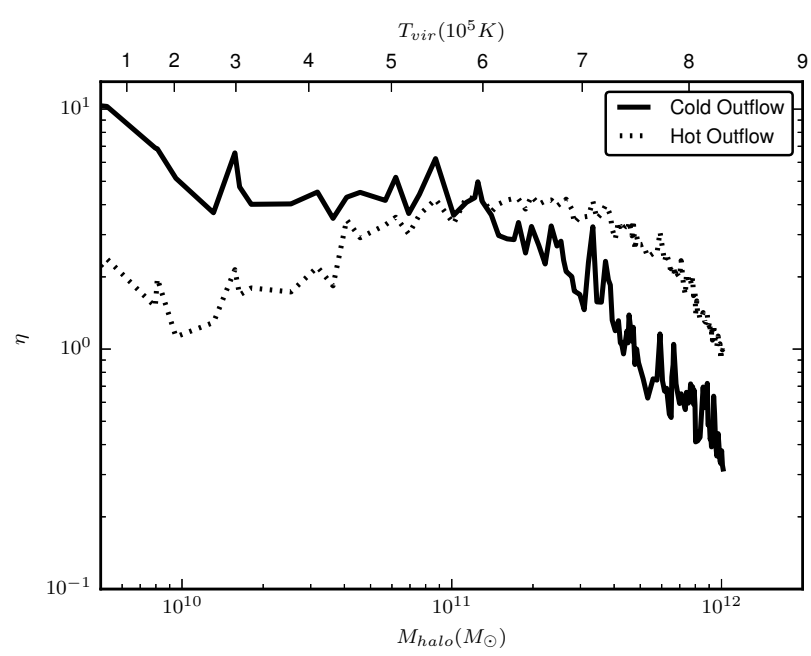

Figure 13. Mean outflow mass loading for the full sample, as a function of halo mass/virial temperature, and split into cold $\left(T<10^{5} \mathrm{~K}\right)$ and hot $\left(T>10^{5} \mathrm{~K}\right)$ components. For low-mass halos, outflows are dominated by relatively cold gas (cooler superbubbles, and entrained material), while heavier halos have primarily hot outflows. The transition occurs at a halo mass of $M_{\text {halo }} \sim 10^{11} \mathrm{M}_{\odot}$, or a virial temperature of $6 \times 10^{5} \mathrm{~K}$.

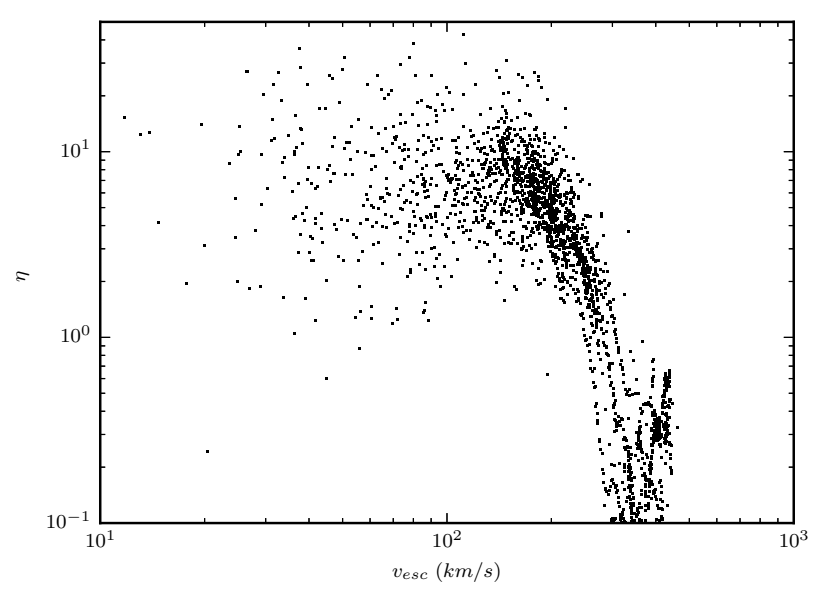

Figure 14. Mean outflow mass loading for the full sample, as a function of the escape velocity on the inner surface of our outflow shell $\left(0.1 R_{v i r}\right)$. Each galaxy is sampled 128 times over its evolution, giving us 2304 points across a range of redshifts. As can be seen, at $v_{\text {esc }} \sim 250 \mathrm{~km} / \mathrm{s}$, the mass loading begins to fall precipitously. This corresponds to the sound speed of solar-metallicity gas at a temperature of $3.5 \times 10^{6} \mathrm{~K}$. This means that for gas at or above this temperature, sound waves alone are able to propel material significantly above the disc, and that kinetic energy losses due to shocks will be minimal. Gas cooler than that will need to be propelled supersonically, and will drive shocks in the CGM.

order of magnitude around that first galaxy. This allows us to probe the expected transition region, where SN feedback begins to fail as a self-regulation mechanism.

The transition between SN regulated galaxies at low mass and AGN regulated galaxies at high mass has been studied observationally in the past decade. Shankar et al. (2006) found that the relation between halo mass and stel- lar mass, $r^{*}$ - band luminosity, or black hole mass all were characterized by double power laws, with breaks at $M_{v i r} \sim 3 \times 10^{11} \quad \mathrm{M}_{\odot}$ or $M_{*} \sim 1 \times 10^{10} \mathrm{M}_{\odot}$. They interpreted these results as the transition between the scalings in SN and AGN regulated galaxies, and constructed a simple analytic model to show the plausibility of this idea. Croton et al. (2006) showed that the most massive galaxies could be produced in a semi-analytic model only when feedback luminosity no longer simply followed the star formation rate (as it would in stellar feedback), but instead scaled with an estimated black-hole accretion lumininosity (as one would expect from AGN feedback).

The most striking feature of this sample of simulated galaxies is the sharp divide between the two identified populations: the well-regulated galaxies and the unregulated galaxies. The well-regulated galaxies are bulgeless and blue, with a surfeit of extra-planar HI gas. The unregulated sample are red and bulge-dominated, with steep rotation curves, with maximum circular velocities as high as $700 \mathrm{~km} / \mathrm{s}$. The members of the well-regulated population have stellar mass fractions below 4\%, while unregulated galaxies have stellar masses all above $6 \%$, with some approaching the cosmic baryon fraction. Clearly, the unregulated galaxies do not match the observed properties of $L *$ galaxies in the nearby universe. Supernovae feedback in these galaxies has failed to prevent runaway bulge growth \& star formation. It has failed because it has failed to efficiently drive winds, which Keller et al. (2015) showed to be essential for the formation of a realistic $L *$ galaxy.

Figure 3 shows that while the unregulated galaxies tend to be more massive than the well-regulated ones, there is some overlap in the $M_{\text {halo }}=8-12 \times 10^{12} \mathrm{M}_{\odot}$ range, where there are both unregulated and well-regulated galaxies. Figure 7 and figure 8 show that the process of runaway star formation happens rapidly, and can begins over a wide range of halo masses. This is further evidence that something beyond simply the mass of the halo that hosts a galaxy is at play in determining whether or not supernova fail to regulate the growth of that galaxy.

A much tighter correlation is seen when looking at the relation between the central baryon mass (essentially, the mass of baryons in the galaxy disc), and the stellar mass fraction. Figures 5 and 9 show that the stellar mass and central mass are tightly correlated, and that once the central mass reaches $\sim 10^{11} \mathrm{M}_{\odot}, \mathrm{SN}$ feedback will begin to fail. This is not particularly surprising, as stars form in the disc, and if SFR occurred at a constant global efficiency, we would expect to see a correlation between the central baryon mass and the stellar mass. However, the two populations show quite different mean star formation efficiencies, with only $\sim 38 \%$ of the well-regulated disc baryons existing in stars compared to $\sim 70 \%$ in the unregulated population. While propelling gas beyond the halo's virial radius will certainly ensure that material does not form stars or collapse through the disc to build a bulge, removing material from the halo isn't necessary to preventing runaway star formation/bulge growth. Material cycling through the CGM, propelled to a few $100 \mathrm{kpc}$ above the disc, can take hundreds of Myr to reaccrete back onto the disc. This means that the increasing escape velocity of the disc is more important to preventing outflows from regulating star formation than an increase in the total halo mass. A more massive halo, with a relatively 
light disc can still have high mass loadings in it's SN-driven outflows, and still have those outflows remove star forming material from the disc for much a galaxy's life.

\subsection{Outflow Scaling Relations and The End of Regulation}

For gas ejected by stellar feedback, the path out of a galaxy disc is fraught. To actually escape the disc, buoyant gas must push through the gas above it. High resolution simulations of disc slices have shown that this means SN occurring higher above or below the disc drive hotter, faster outflows (von Glasow et al. 2013; Sarkar et al. 2015). These results have tended to show that when feedback is deposited randomly, as opposed to at density peaks, stronger outflows can be driven. This is problematic for the simple fact that star formation should be occurring most vigorously in the densest ISM gas, and this should therefore be the site of most feedback deposition. Drift of stars from their natal environment can give some offset between dense gas and where massive stars ultimately end up, but it is unlikely that this is a significant effect for the majority of stars. Governato et al. (2010); von Glasow et al. (2013); Christensen et al. (2015) showed that when SN feedback is clustered, resulting in stronger outflows compared to the same number of SN spread distributed more smoothly throughout the disc. Unfortunately, even these high-resolution, well controlled studies have found significant variation in how wind mass loadings scale with halo mass. The relationship between circular velocity and mass loading has been found to vary both as a function of how feedback energy is injected (von Glasow et al. 2013) and what scaling relationship was used to determine halo mass from of the slice (Creasey et al. (2013) found that using different scaling relations between the halo mass and surface density could give an index $\alpha$ that varies from 2.5 to 4.8). These fully cosmological simulations have the advantage of removing this second source of uncertainty.

The failure of SN to regulate the more massive, discheavy galaxies is ultimately a consequence of the relationship between the efficiency of SN powered outflows and the mass of the halo (or more precisely, the disc). Figures 11 and 12 show that the outflow mass loading is characterized by a broken power-law, with roughly constant mass loadings at low mass, and mass loadings that follow a power-law with negative slope once a critical mass is exceeded. This model for outflow mass loadings is similar to one explored by Font et al. (2011). They found that, in a semi-analytic study of the Milky Way satellites, the best fit for the observed luminosity function was a so-called "saturated feedback" model, where mass loadings were flat at low masses, and decreased as a constant power-law in halos with $v_{c}>65 \mathrm{~km} / \mathrm{s}$ (somewhat lower than the critical value we have found). Further evidence against a single power-law in the $\eta-M$ relation came from recent work by Hou et al. (2015). By combining the analysis of Font et al. (2011) along with more recent MW satellite data, the mass-metallicity relation in local galaxies, and estimates of the redshift of reionization, they found that only a broken power-law for the $\eta-M$ relation can fit all of the observed data. Not only that, their "saturated" model resembles quite closely the fit we have found here, with a flat slope at low masses, and $\eta \propto M_{\text {halo }}^{-1.1}$ for high masses.
(Muratov et al. 2015) found a nearly flat $\eta-M_{\text {halo }}$ relation using the FIRE feedback model, with $\eta \propto M_{\text {halo }}^{-0.35}$.

This relation seems to arise as a result of different $\eta-M$ relations for cold and hot gas. The combination of a steadily decreasing mass loading for cold gas, combined with hot gas mass loadings that peak at $M_{\text {halo }} \sim 5 \times 10^{11} \mathrm{M}_{\odot}$ ultimately result in the broken power-law we see for all outflowing material. In an upcoming study, we will be examining how this relation ultimately arises through detailed examination of ultra high resolution simulations of outflow regions. A detailed study of the hydrodynamics of wind venting should determine the processes by which cold ISM is entrained within the hot outflowing gas, and hopefully explain the origin of the $\eta-M$ relations we have found here.

The results here also show that even in the unregulated population, superbubbles are still able to break out of the galaxy disc (as is shown by the adiabatic evolution of feedback-heated gas seen in figure 6). In order for a superbubble to cool adiabatically by a factor of $\sim 100$, it must be able to expand by that same factor, and only once it has left the galaxy disc is there enough room for it to do this. The failure of SN feedback in the massive, unregulated population is an issue of a drop in efficiency, rather than a complete shutoff, of galactic outflows. The reason the unregulated population exists is that the galaxies in this population spent roughly half of their lifetime above the critical mass in the $\eta-M$ relation, where SN cannot efficiently drive outflows. This has resulted in these galaxies becoming too centrally concentrated, and vastly overproducing stars. Figure 13 and figure 14 show that at higher masses (either for the halo itself, or simply for the interior regions), only the hottest material is able to escape from the disc.

The $\sim 250 \mathrm{~km} / \mathrm{s}$ break in mass loading seen in figure 14 corresponds to the sound speed of relatively hot gas $\left(3.5 \times 10^{6} \mathrm{~K}\right.$ for solar metallicity). Even with no radiative losses whatsoever, if $10^{51} \mathrm{erg}$ is deposited into $1000 \mathrm{M}_{\odot}$ of gas (the specific energy that $\eta=10$ would yield), this gas would have a temperature of $2.5 \times 10^{7} \mathrm{~K}$, and a sound speed of only $\sim 210 \mathrm{~km} / \mathrm{s}$. Thus, it is somewhat unsurprising that the mass-loadings seen here only stay high for escape velocities below $200 \mathrm{~km} / \mathrm{s}$. With mass-loadings falling to unity at escape velocities of almost exactly $300 \mathrm{~km} / \mathrm{s}$, we can infer that the total losses (radiatively and otherwise) between the initial SN explosions and the subsequent outflows are $\sim 80 \%$. The roughly constant outflow rates seen at lower masses/escape velocities imply that a process other than radiative losses is limiting the outflow mass-loadings. The hydrodynamic coupling between hot, outflowing gas and the surrounding medium (the key process that is involved in setting these mass loadings) will be the subject of a subsequent study.

\subsection{Runaway Bulge Growth points to AGN Feedback}

The existence of an unregulated population of galaxies raises the question of what physical mechanism is missing in these simulations. What is important to consider is that this missing physics must be more effective in the high-mass, bulge dominated unregulated population without causing the low mass/well-regulated population to underproduce stars, or disrupt their discs. 
While cosmic rays may be important in certain ISM environments, and may drive the fastest components of galactic winds, there is no known mechanism involved in CR feedback that suggests they become more effective at higher mass. In particular, there is nothing to suggest that the critical mass we see at $M_{\text {halo }} \sim 10^{12} \mathrm{M}_{\odot}$ is in any way related to the efficiency or effectiveness of CR feedback. For the unregulated population, previous results suggest that cosmic rays alone will not be able to produce outflows any more effectively than we have seen SN alone drive, as the mass-loadings they can produce are typically $\ll 10$. Past simulations have also shown that for the mass range where $\mathrm{SN}$ feedback begins to fail to regulate star formation, radiation pressure either fails to prevent overproduction of stars (Aumer et al. 2013), or completely disrupts the thin disc (Roškar et al. 2014), producing "puffy", spherical galaxies with large stellar scale heights, depending on the details of the subgrid model and how that translates into a coupling between the photon fluid and the ISM.

The fact that SN begin to fail at relatively high masses hints that the mechanism that begins to take over in regulating the growth of the galaxy is AGN. It has long been suspected that the high-mass end of galaxy evolution was dominated by AGN feedback from the growth of their SMBHs, and this has been explored heavily in semi-analytic models (Bower et al. 2006; Croton et al. 2006; Shankar et al. 2006). A survey of SDSS galaxies with found that the detectable AGN fraction was essentially zero below $M_{*} \sim 10^{10} \mathrm{M}_{\odot}$, rising to $\sim 50 \%$ by $M_{*} \sim 5 \times 10^{10} \mathrm{M}_{\odot}$, with essentially all galaxies with $M_{*}>5 \times 10^{11} \mathrm{M}_{\odot}$ hosting an AGN (Kauffmann et al. 2003). This transition mass lies exactly where our two populations begin to diverge. No galaxy in our sample with $M_{*}<4 \times 10^{10} \mathrm{M}_{\odot}$ is unregulated, and all but two of the unregulated galaxies have stellar masses above $10^{11} \mathrm{M}_{\odot}$. This is particularly interesting when considering the population of "powerful" AGN in the SDSS peaks at $M_{*} \sim 10^{11} \mathrm{M}_{\odot}$. If these powerful AGN effectively quench star formation within their host galaxies, clamping the stellar mass at or below $10^{11} \mathrm{M}_{\odot}$, this mechanism would put essentially all of our unregulated population back within the Hudson et al. (2015) SMHMR. If these AGN also ejected a significant fraction of the gas within the inner $<1 \mathrm{kpc}$ of the unregulated population, it would also solve the problem of their unrealistically large bulges \& peaked rotation curves.

The correlation between black hole mass and bulge mass (see Kormendy \& Ho (2013) for a review of this evidence) is strong evidence that the central regions co-evolve with with the galaxy (pseudo-)bulge (to first order, this of course must be the case: they are formed from the same material, in roughly the same place). The fact that our unregulated population has grown a much too massive bulge makes AGN feedback an attractive potential resolution. In order to build these massive bulges, angular momentum losses in the gas disc must have been significant, funneling material down towards the center of the disc. This naturally would be a necessary component to fuelling the growth of a SMBH, and powering an AGN. The well-regulated population likely would under produce stars if AGN feedback was a strong effect in those galaxies. Conveniently, the lack of any sort of significant bulge component means that the SMBH within those galaxies would likely be starved of fuel, preventing any significant, sustained AGN feedback. Further evidence for the negligible effect of AGN feedback for these smaller galaxies comes again from the SDSS, where it can be seen that galaxies with $M_{*}<5 \times 10^{10} \mathrm{M}_{\odot}$ contain a powerful AGN in less than $5 \%$ of the cases.

\section{CONCLUSION}

By correctly modelling supernova-driven superbubble feedback, we allow clustered star formation to efficiently drive outflows. This allows SN feedback to produce lower mass galaxies with flat rotation curves, realistic SMHMRs, and small bulges. SN feedback breaks down as a regulator of stellar mass and bulge growth in galaxies with halo masses $>10^{12} \mathrm{M}_{\odot}\left(\right.$ or stellar masses $\left.>4 \times 10^{10} \mathrm{M}_{\odot}\right)$. This breakdown produces a distinct pair of populations. Galaxies below these critical masses have stellar masses within the observed SMHMR, flat rotation curves, and more mass loaded winds over their evolutionary history. When simulated with SN feedback alone, galaxies above this mass have stellar fractions approaching the cosmic baryon fraction, redder stellar populations, steeply peaked rotation curves with maximum circular velocities as high as $700 \mathrm{~km} / \mathrm{s}$, and relatively inefficient outflows. As massive galaxies grow, they move from the well-regulated to unregulated population quickly, typically producing the bulk of their stars in a burst lasting $\leqslant 1$ Gyr, after which star formation slows simply due to a dearth of gas to form stars out of.

The cause for this transition is the relation between the mass loading of superbubble outflows $\eta$ and the halo or disc mass. A critical value exists for both of these masses, where mass loadings begin to drop steeply as mass increases, reducing the ability of SN to regulate the SFR and baryon content of the galaxy. Once the escape velocity from the inner $0.1 R_{v i r}$ exceeds $\sim 250 \mathrm{~km} \mathrm{~s}^{-1}$, mass loadings fall rapidly to $>1$. A simple broken power-law fit describes the relation between the outflow mass loading and both the halo mass and disc mass. For disc mass below $10^{10} \mathrm{M}_{\odot}$ and halo mass below $2 \times 10^{11} \mathrm{M}_{\odot}$, outflow mass loadings are approximately constant, with $\eta \sim 8$.

SN feedback begins to fail at exactly the mass range that strong AGN are observationally detected. This coincides with the runaway growth of massive stellar bulges in the unregulated population. The associated black hole feeding and nuclear activity should not only regulate bulge growth but also the total baryonic distribution and star formation for galaxies at the high mass end. Up to this mass, supernova-driven superbubbles alone can regulate the baryonic content and star formation in galaxies.

\section{ACKNOWLEDGEMENTS}

The analysis was performed using the pynbody package (http://pynbody.github.io/, (Pontzen et al. 2013)). We thank Alyson Brooks, Fabio Governato, Tom Quinn, and John Kormendy for useful conversations regarding this paper. The simulations were performed on the clusters hosted on SCINET, part of ComputeCanada. We greatly appreciate the contributions of these computing allocations. We also thank NSERC for funding supporting this research. 


\section{REFERENCES}

Agertz O., Kravtsov A. V., 2015, ApJ, 804, 18

Aumer M., White S. D. M., Naab T., Scannapieco C., 2013, MNRAS, 434, 3142

Bailin J., Stinson G., Couchman H., Harris W. E., Wadsley J., Shen S., 2010, ApJ, 715, 194

Behroozi P. S., Wechsler R. H., Conroy C., 2013, ApJ, 770, 57

Booth C. M., Agertz O., Kravtsov A. V., Gnedin N. Y., 2013, ApJ, 777, L16

Bower R. G., Benson A. J., Malbon R., Helly J. C., Frenk C. S., Baugh C. M., Cole S., Lacey C. G., 2006, MNRAS, 370, 645

Breitschwerdt D., McKenzie J. F., Voelk H. J., 1991, A\&A, 245, 79

Bullock J. S., Dekel A., Kolatt T. S., Kravtsov A. V., Klypin A. A., Porciani C., Primack J. R., 2001, ApJ, 555, 240

Chabrier G., 2003, PASP, 115, 763

Christensen C. R., Davé R., Governato F., Pontzen A., Brooks A., Munshi F., Quinn T., Wadsley J., 2015, preprint, (arXiv:1508.00007)

Chu Y.-H., Kennicutt Jr. R. C., 1994, ApJ, 425, 720

Cole S., Lacey C. G., Baugh C. M., Frenk C. S., 2000, MNRAS, 319,168

Crain R. A., et al., 2015, MNRAS, 450, 1937

Creasey P., Theuns T., Bower R. G., 2013, MNRAS, 429, 1922

Croton D. J., et al., 2006, MNRAS, 365, 11

Dale J. E., Ercolano B., Bonnell I. A., 2012, MNRAS, 424, 377

Davé R., Hellsten U., Hernquist L., Katz N., Weinberg D. H., 1998, ApJ, 509, 661

Dekel A., Silk J., 1986, ApJ, 303, 39

Dubois Y., et al., 2014, MNRAS, 444, 1453

Efstathiou G., 1992, MNRAS, 256, 43P

Erb D. K., 2008, ApJ, 674, 151

Everett J. E., Zweibel E. G., Benjamin R. A., McCammon D., Rocks L., Gallagher III J. S., 2008, ApJ, 674, 258

Finlator K., Davé R., 2008, MNRAS, 385, 2181

Font A. S., et al., 2011, MNRAS, 417, 1260

Girichidis P., et al., 2015, preprint, (arXiv: 1509.07247)

Governato F., et al., 2010, Nature, 463, 203

Gupta A., Mathur S., Krongold Y., Nicastro F., Galeazzi M., 2012, ApJ, 756, L8

Hahn O., Martizzi D., Wu H.-Y., Evrard A. E., Teyssier R., Wechsler R. H., 2015, preprint, (arXiv:1509.04289)

Heckman T. M., Armus L., Miley G. K., 1987, AJ, 93, 276

Hopkins P. F., 2013, MNRAS, 428, 2840

Hopkins P. F., Kereš D., Oñorbe J., Faucher-Giguère C.-A., Quataert E., Murray N., Bullock J. S., 2014, MNRAS, 445,581

Hou J., Frenk C. S., Lacey C. G., Bose S., 2015, preprint, (arXiv:1512.04595)

Hudson M. J., et al., 2015, MNRAS, 447, 298

Ipavich F. M., 1975, ApJ, 196, 107

Jubelgas M., Springel V., Enßlin T., Pfrommer C., 2008, A\&A, 481,33

Kauffmann G., et al., 2003, MNRAS, 346, 1055

Keller B. W., Wadsley J., Benincasa S. M., Couchman H. M. P., 2014, MNRAS, 442, 3013

Keller B. W., Wadsley J., Couchman H. M. P., 2015, MNRAS, 453, 3499

Kormendy J., Ho L. C., 2013, ARAA, 51, 511

Krumholz M. R., Matzner C. D., 2009, ApJ, 703, 1352

Krumholz M. R., Thompson T. A., 2013, MNRAS, 434, 2329

Kunth D., Mas-Hesse J. M., Terlevich E., Terlevich R., Lequeux J., Fall S. M., 1998, A\&A, 334, 11

Larson R. B., 1974, MNRAS, 169, 229

Leitherer C., et al., 1999, ApJS, 123, 3

Leroy A. K., et al., 2015, ApJ, 814, 83

Lynds C. R., Sandage A. R., 1963, ApJ, 137, 1005
Mac Low M.-M., McCray R., 1988, ApJ, 324, 776

Magorrian J., et al., 1998, AJ, 115, 2285

Marasco A., Fraternali F., Binney J. J., 2012, MNRAS, 419, 1107

Marigo P., Girardi L., Bressan A., Groenewegen M. A. T., Silva L., Granato G. L., 2008, A\&A, 482, 883

Martin C. L., Shapley A. E., Coil A. L., Kornei K. A., Bundy K., Weiner B. J., Noeske K. G., Schiminovich D., 2012, ApJ, 760,127

Mathews W. G., Baker J. C., 1971, ApJ, 170, 241

Meyer L., et al., 2012, Science, 338, 84

Morganti R., Oosterloo T. A., Emonts B. H. C., van der Hulst J. M., Tadhunter C. N., 2003, ApJ, 593, L69

Moster B. P., Naab T., White S. D. M., 2013, MNRAS, 428, 3121

Muratov A. L., Keres D., Faucher-Giguere C.-A., Hopkins P. F., Quataert E., Murray N., 2015, preprint, (arXiv:1501.03155)

Murray N., Quataert E., Thompson T. A., 2005, ApJ, 618, 569

Murray N., Ménard B., Thompson T. A., 2011, ApJ, 735, 66

Nickerson S., Stinson G., Couchman H. M. P., Bailin J., Wadsley J., 2011, MNRAS, 415, 257

Nickerson S., Stinson G., Couchman H. M. P., Bailin J., Wadsley J., 2013, MNRAS, 429, 452

Peeples M. S., Shankar F., 2011, MNRAS, 417, 2962

Pontzen A., Roškar R., Stinson G. S., Woods R., Reed D. M., Coles J., Quinn T. R., 2013, pynbody: Astrophysics Simulation Analysis for Python

Ritchie B. W., Thomas P. A., 2001, MNRAS, 323, 743

Rogers H., Pittard J. M., 2013, MNRAS, 431, 1337

Roškar R., Teyssier R., Agertz O., Wetzstein M., Moore B., 2014, MNRAS, 444, 2837

Salem M., Bryan G. L., 2014, MNRAS, 437, 3312

Sarkar K. C., Nath B. B., Sharma P., Shchekinov Y., 2015, MNRAS, 448, 328

Schaye J., et al., 2015, MNRAS, 446, 521

Shankar F., Lapi A., Salucci P., De Zotti G., Danese L., 2006, ApJ, 643, 14

Shen S., Wadsley J., Stinson G., 2010, MNRAS, 407, 1581

Sijacki D., Vogelsberger M., Genel S., Springel V., Torrey P., Snyder G. F., Nelson D., Hernquist L., 2015, MNRAS, 452, 575

Socrates A., Davis S. W., Ramirez-Ruiz E., 2008, ApJ, 687, 202

Songaila A., Cowie L. L., 1996, AJ, 112, 335

Stark A. A., Carlson E. R., 1984, ApJ, 279, 122

Stinson G., Seth A., Katz N., Wadsley J., Governato F., Quinn T., 2006, MNRAS, 373, 1074

Stinson G. S., Bailin J., Couchman H., Wadsley J., Shen S., Nickerson S., Brook C., Quinn T., 2010, MNRAS, 408, 812

Stinson G. S., Brook C., Macciò A. V., Wadsley J., Quinn T. R., Couchman H. M. P., 2013, MNRAS, 428, 129

Tegmark M., Silk J., Evrard A., 1993, ApJ, 417, 54

Veilleux S., Cecil G., Bland-Hawthorn J., 2005, ARAA, 43, 769

Wang L., Dutton A. A., Stinson G. S., Macciò A. V., Penzo C., Kang X., Keller B. W., Wadsley J., 2015, MNRAS, 454, 83

Weiner B. J., et al., 2009, ApJ, 692, 187

Woods R. M., Wadsley J., Couchman H. M. P., Stinson G., Shen S., 2014, MNRAS, 442, 732

Zweibel E. G., McKee C. F., 1995, ApJ, 439, 779

van den Bosch F. C., 2001, MNRAS, 327, 1334

von Glasow W., Krause M. G. H., Sommer-Larsen J., Burkert A., 2013, MNRAS, 434, 1151 